\title{
Penser liquide. Vers une esthétique écologique (Morton, Castaing-Taylor \& Paravel)
}

\section{Liquid thinking. Towards an ecological aesthetic (Morton, Castaing-}

\author{
Taylor \& Paravel)
}

\author{
Sophie Lécole Solnychkine ${ }^{1}$ \\ ${ }^{1}$ LARA-SEPPIA, Université Toulouse - Jean-Jaurès
}

\begin{abstract}
RÉSUMÉ. Ce texte propose, au regard des notions de «maillage » (mesh) et d' « étranges étrangers » (strange strangers) développées par Timothy Morton, de questionner les moyens esthétiques du cinéma, lorsqu'il s'attèle à saisir des phénomènes écologiques dont les arts paysagers issus du modèle pictural classique achoppent à former une image pertinente. L'étude de Leviathan (Castaing-Taylor \& Paravel, 2012) sera l'occasion de questionner le paradoxe qui consiste à vouloir penser conjointement écologie, conscience (et expérience) du milieu et persistance du modèle paysager.

ABSTRACT. Relying on Timothy Morton's concepts of "mesh" and "strange strangers", this article aims to question the aesthetic means of cinema when it strives to figure and represent ecological phenomena which landscape arts based on the classical pictorial model fail to fashion into a relevant image. This study of Leviathan (Castaing-Taylor \& Paravel, 2012) questions the paradox of wanting to think ecology, consciousness (and experience) of milieu, and the persitence of the landscape scheme together.

MOTS-CLÉS. Esthétique, cinéma, théorie du cinéma, ethnographie, écologie, pensée écologique, maillage, étrange étranger, Timothy Morton, art et écologie, pêche au gros, paysage, modèle paysager, environnement, milieu.

KEYWORDS. Aesthetics, cinema, film theory, ethnography, ecology, ecological thought, mesh, strange stranger, Timothy Morton, art and ecology, deep sea fishing, landscape, landscape scheme, environment, milieu.
\end{abstract}

Leviathan est un film réalisé en 2012 par deux ethnographes-réalisateurs, la Suisse Véréna Paravel et le Britannique Lucien Castaing-Taylor. Le film a été produit dans le cadre du Sensory Ethnography Lab de l'Université de Harvard, dont Castaing-Taylor est le fondateur. Créé en 2006, le Sensory Ethnography Lab se situe au croisement des départements d'Anthropologie et d'Études visuelles et environnementales, et a pour mission de produire des objets filmiques ${ }^{1}$ qui pensent conjointement forme et contenu, en associant pratiques audiovisuelles expérimentales, réflexion sur les formes esthétiques et démarche ethnographique rigoureuse, et qui, à partir de là, repensent ce que peut être le film ethnographique, voire le film documentaire en son ensemble. Comme s'en est expliqué Lucien Castaing-Taylor dans le New York Times, le Lab «prend au sérieux l'ethnographie. Ce n'est pas comme si vous pouviez faire de l'ethnographie en une visite de deux jours quelque part. Mais il prend également le sensoriel au sérieux. La plupart des écrits anthropologiques et des films,

\footnotetext{
${ }^{1}$ Parmi les autres productions du Sensory Ethnography Lab, on peut mentionner des films comme Manakamana (Stephanie Spray et Pacho Velez, 2013) ou People's Park (J.P. Sniadecki et Libbie D. Cohn, 2012) ; un travail d'enquête sonore comme Materiel Recovery Facility (Ernst Karel, 2011) ; Single Stream, une installation de Pavel Wojtasik, Toby Lee et Ernst Karel qui explore le traitement industriel des déchets. Un journal multimédia, Sensate, Journal for experiments in Critical Media Practice, a été créé, qui propose des œuvres en ligne, au carrefour des pratiques artistiques et de la recherche en sciences humaines.
} 
à l'exception de quelques œuvres remarquables, sont tellement dénués d'expérience émotionnelle ou sensible ». Enfin, « il prend au sérieux ce que l'art peut faire ${ }^{2} »$.

Célébré pour son originalité, Leviathan relève d'un dispositif filmique tout à fait particulier, qui s'inscrit à plein dans le questionnement des moyens esthétiques du cinéma, et plus largement des images animées, lorsque celles-ci s'attèlent à la tâche de saisir des phénomènes écologiques dont les arts paysagers issus du modèle pictural classique achoppent à former une image pertinente. L'étude de ce film sera pour moi l'occasion de questionner le paradoxe qui consiste à vouloir penser conjointement écologie, conscience (et expérience) du milieu et persistance du modèle paysager.

$\mathrm{Si}$, comme le pense Timothy Morton, dont j'explorerai les perspectives dans ce texte, penser écologique consiste à se débarrasser des réquisits de l'idée de Nature (mirage de l'origine, fantasme d'un état d'harmonie perdu, illusion de l'authenticité des êtres et des milieux), force est de constater que ce projet demande, à terme, de renoncer au paysage en tant que mode d'ordonnancement du regard, c'est-à-dire comme puissance de hiérarchisation du réel (le «pittoresque » désigne littéralement « ce qui est digne d'être peint»; le paysage est conçu comme «vitrine » de l'idée de Nature). Alors, à suivre les concepts proposés par Morton, c'est à la saisie du maillage ${ }^{3}$ (mesh) qu'il faut s'atteler, dont les arts peuvent, à leur manière, proposer des approches stimulantes. Le maillage désigne, chez Morton, l'interconnectivité de toutes choses, humaines comme non-humaines, vivantes comme non vivantes. Le maillage, tel que le pense Morton, n'a ni centre, ni bords. Il est incommensurable. Il implique de "penser grand», dans l'espace comme dans le temps, ce qui mène à découvrir la puissance d'estrangement du regard écologique, qui conduit à voir la Terre depuis l'espace, comme une sphère qui flotte. Penser grand provoque notre désorientation, nous engage à renoncer à la possibilité de discerner un premier plan d'un arrière-plan, et nous invite à rencontrer, aux jointures du maillage, une «intimité radicale ${ }^{4}$ » avec d'autres êtres, sensibles ou non, vivants ou non, qui sont, tout comme nous, les produits inquiétants de mutations et d'événements catastrophiques, saisis dans des échelles d'espaces et de temps incommensurables : les « étranges étrangers » (strange strangers).

Dérivée de l'«arrivant» de Derrida et d'Anne Dufourmantelle (De l'hospitalité, Calmann-Lévy, 1997), terme qui désignait l'arrivant ultime auquel on doit fournir l'ultime hospitalité, la notion d'étrange étranger de Morton est difficile à cerner. Elle désigne, dans la perspective du maillage, «les formes du vivant auxquelles nous nous trouvons connectés ${ }^{5}$ », des formes avec lesquelles nous possédons une intimité inquiétante et monstrueuse, qui questionne nos certitudes au sujet de la notion même de personne, lorsque, par exemple, la théorie de l'endosymbiose nous apprend que vivent en nous, tapies dans nos cellules, des bactéries originelles, des procaryotes datant de

\footnotetext{
${ }^{2}$ Cité par Marie LECHNER, in « À Harvard, des documentaires qui explorent les sens », Libération, [En ligne] URL = [https://next.liberation.fr/cinema/2013/08/28/a-harvard-des-documentaires-qui-explorent-les-sens_927684], 28 août 2013, consulté le 14 mai 2019.

3 "Le "maillage" renvoie aux trous dans un réseau et aux fils qui les relient. Il évoque à la fois la solidité et la finesse. On l'emploie en biologie, en mathématiques et en ingénierie, dans le textile et en informatique - pensez aux collants et au graphisme, au métal et au tissu. "Mesh", le "maillage", a des liens étymologiques avec masque et masse, qui évoquent à la fois la densité et l'illusion. Par extension, "mesh" peut signifier "une situation complexe ou une série d'événements dans laquelle est prise une personne; une concaténation de forces ou de circonstances contraignantes ou restrictives; un piège". En d'autres termes, il est parfait. ", in Timothy MORTON, La pensée écologique, trad. (anglais) Cécile Wajsbrot, Paris, Éditions Zulma, coll. « Zulma essais », 2019 [2010], p. 55-56.
}

${ }^{4}$ Ibid., p. 23.

${ }^{5}$ Ibid., p. 38. 
l'Archéen anaérobie (entre 2,5 et 4,5 milliards d'années), responsables du premier désastre écologique, celui de la création de l'oxygène, et réfugiées au plus intime de nos organismes. De nombreux éléments en nous proviennent de mutations antérieures, d'états très anciens d'êtres inconnus qui ont figuré parmi nos «ancêtres » : le pavillon de nos oreilles est un vestige d'oreille «animale » ayant la capacité de se dresser, tandis que nos nerfs crâniens sont issus des arcs branchiaux des poissons. L'étrange étranger, c'est nous, c'est la part de nous qui échappe à notre perception, c'est le produit de l'évolution que l'on contemplerait à vitesse tellement accélérée que les êtres qui nous semblent familiers ne seraient plus qu' « un flux de morphoses inquiétantes ${ }^{6}$ », liées à la dimension mutagène de l'évolution, qui laisse sans dessein préconçu l'ADN poursuivre son évolution aléatoire.

Dernier avatar, l'étrange étranger peut aussi désigner l'environnement : refusant la perspective adéquationniste de l'évolution, et la croyance en une "essentialité » des environnements auxquels les espèces s'adapteraient, Morton considère ce que l'on pourrait désigner comme une forme de viscosité, de symbiogénèse, de coévolution entre les environnements et les êtres qui les peuplent :

«Puisque les étranges étrangers, nous dit Morton, sont tout autour de nous, considérons la nature de ce "tout autour". Les environnements sont constitués d'étranges étrangers. Le phénotype produit par le génotype génétique inclut l'environnement, de même qu'un barrage de castors ou un nid de souris. L'environnement co-évolue avec les organismes. Le monde a l'aspect qu'il a en raison des formes du vivant. L'environnement n'existe pas "en dehors" d'elles ${ }^{7}$ ».

Enfin, « il n'y aurait pas de maillage s'il n'y avait pas d'étranges étrangers. Le maillage n'est pas un arrière-plan sur lequel apparaît un étrange étranger. C'est l'enchevêtrement de tous les étrangers ${ }^{8} \gg$.

C'est donc en usant de ces outils que je propose d'étudier le film de Véréna Paravel et Lucien Castaing-Taylor, autour de 5 entrées que j'ai déclinées comme suit : une politique du cadre ; plonger dans les images; une esthétique au-delà de l'humain; une éthique du sonore ; une écologie des formes. Enfin, en guise de conclusion, je reviendrai sur la fonction d'un art « écologique».

\section{Une politique du cadre}

J'examinerai d'abord comment, dans Leviathan, le cadre est obtenu (en termes de dispositif) ; ensuite ce que l'on y trouve (en termes de figuration) - bien sûr, on verra comment ces deux régimes d'attention sont indissociables dans le projet du film.

Leviathan use d'un dispositif tout à fait particulier, dont on ne peut que louer à la fois la cohérence qu'il engage entre le fond et la forme du film, et l'extraordinaire saisie esthétique du maillage qu'il orchestre, pour peu que l'on suive la terminologie mortonienne. S'attachant à investiguer l'expérience de la pêche professionnelle en mer, le film est tourné sur un chalutier de 25 mètres, en Atlantique Nord, au large des côtes de New Bedford en Nouvelle-Angleterre (ancienne capitale mondiale de la chasse à la baleine, celle-là même qui a inspiré à Melville son Moby Dick). Le film relève d'un dispositif immersif : usant de 10 GoPros sanglées sur les bras, les torses, les

\footnotetext{
${ }^{6}$ Ibid., p. 80.

${ }^{7}$ Ibid., p. 91.

${ }^{8}$ Ibid., p. 85.
} 
têtes, les réalisateurs ont le souci d'impliquer physiquement les pêcheurs, mais aussi les animaux et les puissances élémentales. Ainsi, comme le note Cyril Neyrat, "ils abandonnent le contrôle de leurs cadres aux mouvements aléatoires des hommes et des éléments »: «[...] au lieu d'employer les dernières technologies à un surcroît de maîtrise du visible et de contrôle panoptique de la réalité, ils ont saisi la chance qu'elles offraient de partager cette maîtrise avec le monde, de mettre en œuvre [...] le partage démocratique du sensible (Jacques Rancière) ${ }^{9} »$. Ainsi, comme le confient les deux réalisateurs à Jean-Michel Frodon, « on s'est approchés des hommes au point de les harnacher de nos caméras, et pourtant ce n'est pas un film sur les hommes. [...] Ici, ils font simplement partie de la nature. Ils sont à leur place, dans un cosmos plus large [...], une sphère écologique qui les dépasse $^{10} \gg$.

Paradoxalement, l'ensemble des images du film donne la sensation d'une caméra stable - au sens où elle ne tangue pas démesurément au gré des flots -, mais par contre extraordinairement mobile, vivace, furetant dans le champ, suivant rapidement, parfois jusqu'au vertige, les objets par de rapides mouvements d'appareil, dont on éprouve, plus que l'intention de vision, l'organicité. Fixées sur le corps des navigateurs, les 10 GoPros «[permettent] une façon de filmer avec le corps tout entier [je souligne] ${ }^{11} »$, qui engage une façon très particulière de voir. Fortement présente, la sensation que les images sont dictées par le mouvement des corps détermine un régime esthétique très particulier : l'impression de ne pas voir par les yeux, mais de voir par le corps, par la présence, rendue voyante, de toute la kinésphère ${ }^{12}$.

Comme l'expliquent les réalisateurs, « cette façon très physique de filmer rend compte de la façon dont nous étions aux prises avec la force des éléments. [...] Il n’y a que ces caméras qui répondaient à cette exigence d'être si corporellement engagés avec les êtres, les éléments, les machines ${ }^{13} \gg$. Ayant perdu, lors de tempêtes en mer (qui font donc partie intégrante du dispositif du film, pour peu que l'on s'y intéresse dans l'approche écologique qui est la nôtre), les caméras avec lesquelles ils étaient habitués à travailler, les réalisateurs expliquent comment, a posteriori, cette avarie leur est apparue comme providentielle, puisque les images que leur donnaient ces caméras étaient finalement plates et conventionnelles comparativement aux images qu'ils obtiendraient in fine. L'usage des GoPros, desquelles relèvent pratiquement toutes les images du film ${ }^{14}$, génère des images heurtées, brouillées, qui frôlent l'abstraction, ou détonent par leur picturalité : « des images qui accentuent - sûrement parce que leur qualité est compromise - le mélange de beauté et d'horreur du carnage organisé lors de ces campagnes de pêche ${ }^{15}$ ». Pour leur donner encore la parole : «On a

\footnotetext{
${ }^{9}$ Cyril NEYRAT, « Le sang des poissons, la beauté du monstre ", in Revue Independencia [En ligne] URL = [http://www.independenciarevue.net/s/spip.php?article744], consulté le 21 mai 2019.

${ }^{10}$ Jean-Michel FRODON, « Les visions sans précédent de Leviathan », Entretien avec Véréna Paravel et Lucien Castaing-Taylor [En ligne], in Slate, août 2013, URL = [http://www.slate.fr/grand-format/leviathan], consulté le 11 août 2019.
}
${ }^{11}$ La citation est tirée d'un entretien intitulé « Leviathan, un film fou signé Véréna Paravel et Lucien Castaing-Taylor », [En ligne], $\mathrm{URL}=$ [http://leblogdocumentaire.fr/leviathan-un-documentaire-de-verena-paravel-et-lucien-castaing-taylor/], consulté le 14 mai 2019.

12 Définie par le chorégraphe et théoricien de la danse Rudolf Laban, la notion de kinésphère désigne une sphère imaginaire englobant l'espace accessible par le mouvement d'un être, soit l'espace de proximité qui s'étend autour d'une personne et qui est déterminé par l'étendue de ses gestes, jusqu'à l'extrémité de ses membres tendus dans toutes les directions.

13 « Leviathan, un film fou signé Véréna Paravel et Lucien Castaing-Taylor », Ibid.

${ }^{14}$ Une caméra DSLR complète simplement la batterie de GoPros.

15 « Leviathan, un film fou signé Véréna Paravel et Lucien Castaing-Taylor », Ibid. 
voulu créer une esthétique qui déstabilise notre habitude de regarder le monde d'un seul point de vue, celui des humains qui font des films. On s'est approchés le plus possible, on s'est accrochés à tous les recoins, à tous les corps pour créer un cinéma physique, ou plus physique que discursif, moins appliqué à imposer du sens, et soucieux de re-atteler l'humanité à notre nature bestiale, la juxtaposer à la bestialité des êtres non-humains, les autres espèces, et la nature ${ }^{16}{ }$.

\section{Plonger dans les images}

Ouverture. Le film débute par des images abstraites, sombres, dévorées de façon intermittente par des flashes de couleur, qui illuminent des surfaces que l'œil peine à assigner. Le son, pour une fois fait rare au cinéma - plus signifiant que l'image, indique que des matériaux métalliques sont manipulés, qu'ils s'entrechoquent, qu'ils produisent par frottement toute une famille de bruits qui peuple l'espace. Tout à coup, étoilant l'écran, une giclée d'eau vient prendre la place d'une étincelle. Puis, au bout d'un long moment, ces perceptions désordonnées s'organisent peu à peu. Des formes émergent du magma incertain de la bande-image. Une chaîne à gros maillons, rongée par la corrosion. Un casier métallique de grande dimension hissé depuis le fond d'une masse d'eau sans autre limite que les bords du cadre. Des fragments de silhouettes humaines, revêtues de cirés jaunes ou oranges ; des mains gantées de bleu ou de rouge entrent sporadiquement dans le champ et se saisissent d'objets. Le miroitement de lumières électriques sur la surface saline de l'océan. Des paquets d'écume, la crête des vagues qui se découpe dans la lumière électrique du bateau. Un coin d'aurore ou de crépuscule qui déchire le ciel uniformément noir. Des cris étouffés, humains ou animaux, à demi masqués par le sifflement de la houle atlantique et le ressac des vagues qui s'écrasent contre la coque. La masse mouvante et terrifiante de l'océan dans la nuit, éclairée de vert, qui semble si proche du pont qu'elle pourrait, à tout instant, le submerger ; d'autant que l'angle de prise de vue, gîtant par-dessus bord, penche fortement à l'oblique. La remontée du filet, les immenses casiers au sol, le treuil qui arrache la masse vivante et remuante des profondeurs de l'océan, les gouttes d'eau omniprésentes, les tâches sur l'objectif de la caméra qui déforment les contours des objets lorsqu'ils sont reconnaissables, ne laissant à l'œil aucun répit, le ruissellement continu, le cri des machines et les ordres des hommes, les mouettes - en vol stationnaire au-dessus de l'endroit d'où émergent les filets - qui constellent l'obscurité de la nuit de tâches claires, à la limite de la lisibilité, enfin, les poissons, beaucoup de poissons, que des mains gantées saisissent, que des doigts recouverts de plastique étêtent au couteau. Tout se couvre progressivement de sang, d'un badigeon d'entrailles de poisson. Partout, l'eau ruisselle sur l'objectif, créant des zones de trouble, des taches colorées qui fondent ensemble les objets du monde.

\section{Une esthétique au-delà de l'humain}

Leviathan surprend parce qu'il conjugue des points de vue multiples : humains comme nonhumains, vivants comme inertes. Il nous place en plein cœur de cette interconnexion, qui est aussi une coexistence, décrite par Timothy Morton sous le terme de maillage. En adoptant d'autres ordres de perception que celui des humains, d'autres régimes de monstration que ceux auxquels le cinéma nous a habitués, Leviathan désanthropocentrise l'approche documentaire. Les réalisateurs, dans un entretien accordé à Jérôme Momcilovic, parlent d'ailleurs à dessein de «recontextualiser » leur

\footnotetext{
${ }^{16}$ Jean-Michel Frodon, op. cit.
} 
représentation de l'humain et de son travail de subsistance «dans une dimension écologique et cosmique plus large $»^{17}$.

Les séquences subaquatiques sont à ce titre édifiantes. D'abord signalées par le son (puisque ce sont les seuls moments où s'estompe le bruit omniprésent des moteurs), elles montrent des enchaînements de textures, des formes instables tracées par la masse mouvante de l'océan, zébrées de traits convulsifs constitués par le trajet des bulles d'air et la réflexion de la lumière [fig. 1]. Images constituées de courants d'eau, mues par le ressac et tiraillant l'œil en tous sens, elles nous confrontent, de loin en loin, au rythme heurté du montage, à une algue en suspension, qui, en constituant une façon de premier plan, permet au spectateur de goûter la profondeur de l'eau, sa masse, son épaisseur, son opacité : de se positionner, pour un fragile instant, sous la surface [fig. 2]. En outre, ces séquences posent la question de leur place dans l'économie plus large du récit audiovisuel, et de leur autonomie par rapport à celles qui les précèdent. On y coule comme bascule le filet dans la mer, comme glisse le poisson dans les courants. Qu'y voit-on ? Et surtout, qu'est-on lorsque l'on perçoit cela ? Un poisson ? Une coquille Saint-Jacques ? Une étoile de mer ? Le filet ? Le filin qui le meut ? Tout cela à la fois ?

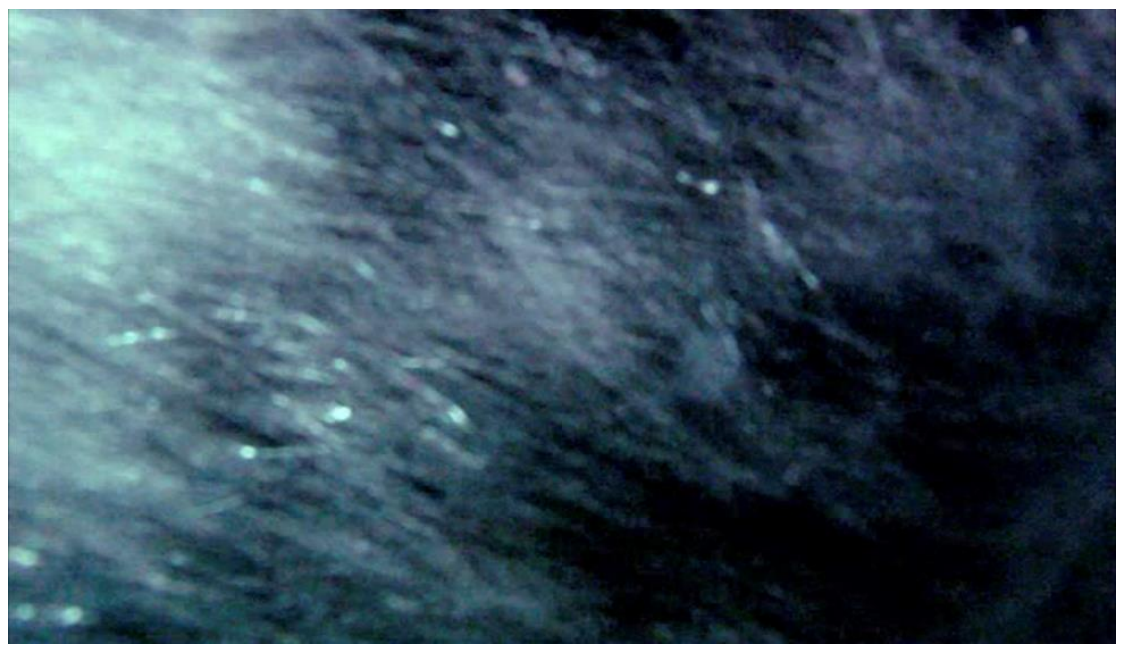

Fig. 1. Leviathan, Véréna Paravel et Lucien Castaing-Taylor, 2012, photogramme du film.

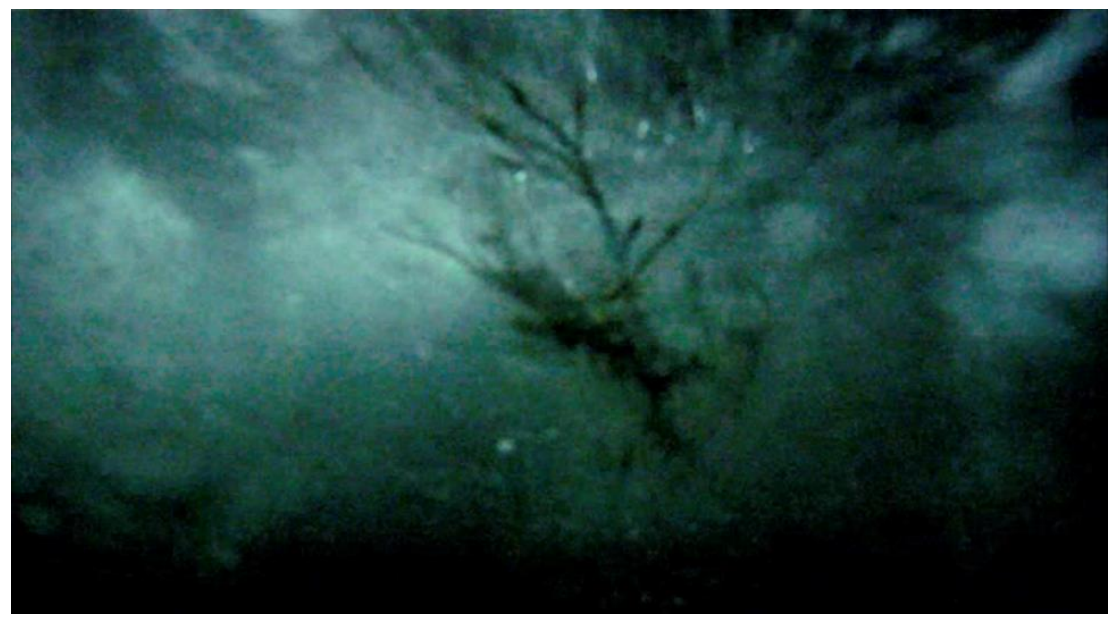

Fig. 2. Leviathan, Véréna Paravel et Lucien Castaing-Taylor, 2012, photogramme du film.

\footnotetext{
17 Jérôme MoMCILOVIC, « Lucien Castaing-Taylor et Verena Paravel : Into the Abyss », Chronic'Art, [En ligne], URL = [https://www.chronicart.com/cinema/lucien-castaing-taylor-et-verena-paravel-into-the-abyss/], août 2013, consulté le 11 août 2019.
} 
Plus tard, c'est une séquence présentant des images noires traversées d'infimes formes blanches qui interroge le regard. Quelle est la nature de ce que nous voyons ? D'où voyons-nous ? Est-on au fond de l'eau ? Ou bien sur le pont, enfouis au plus sombre du tas inerte de poissons ? Ou encore, avons-nous, d'un saut optique d'échelle, plongé au plus infime de la matière, baignant dans le repli d'une quelconque vie moléculaire ? Se pose ici la question de la possibilité de regards non-humains, signes d'une esthétique au-delà de l'humain.

Sur le pont du bateau, d'abord dans un casier à poissons, puis plus tard au fond d'un seau, l'appareil optique incarne longuement l'un de ces corps à l'agonie, épousant le va-et-vient des poissons morts ou en train de s'asphyxier [fig. 3 et 4]. Séparés de notre milieu, étranges étrangers, nous glissons à fond de bac, dans un résidu d'eau saumâtre mêlée de sang, emportés d'avant en arrière par les mouvements du bateau, lui-même mu par le flux incessant de l'océan et le rythme des turbines qui l'arrachent à l'inertie de la masse liquide. Ritournelle visuelle des corps flottant entre deux eaux, on heurte les poissons du regard ; ils s'éloignent, reviennent frapper l'objectif de la flaccidité de leur chair, imageant le «moment d'avant» d'une nature morte halieutique, la Raie d'un Chardin ou d'un Soutine. Depuis les soubresauts d'agonie de cette masse grouillante, frappent l'œil la richesse chromatique des nageoires brillantes et des fluides poisseux, les découpes subtiles des barbillons et des ouïes, les textures des écailles, des globes oculaires, des branchies.

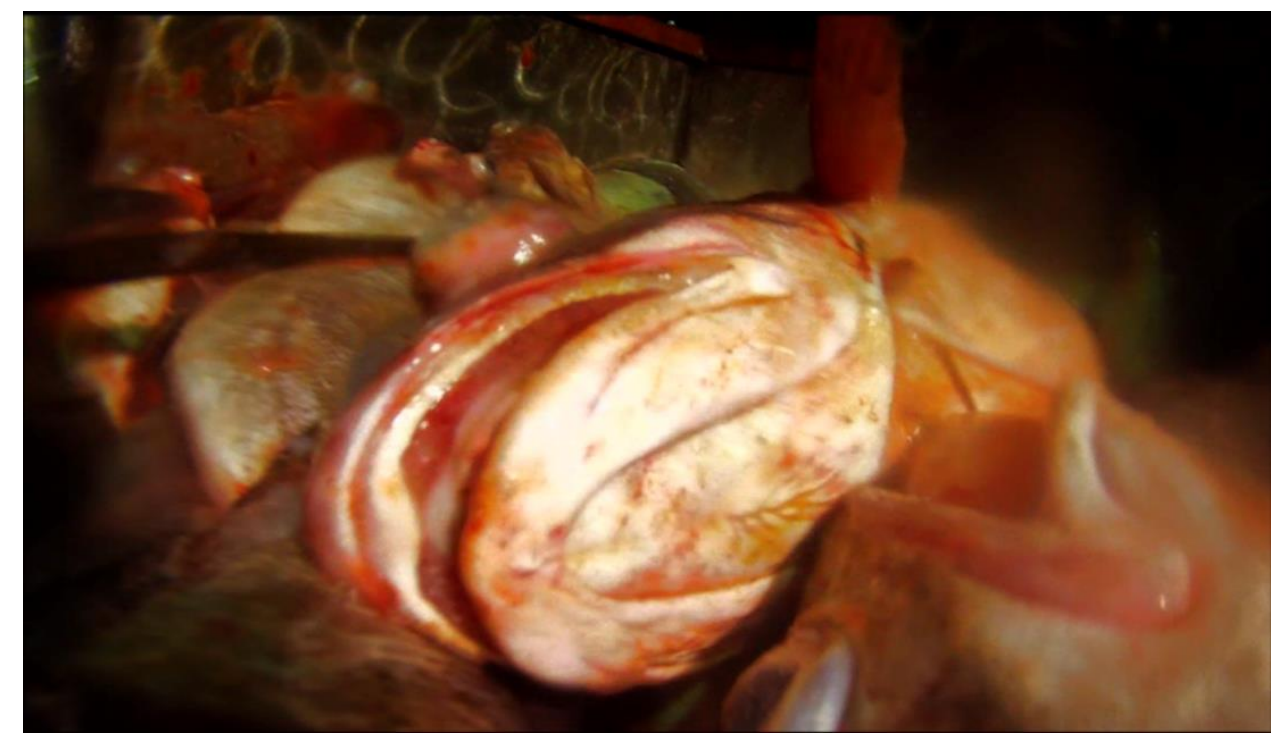

Fig. 3. Leviathan, Véréna Paravel et Lucien Castaing-Taylor, 2012, photogramme du film.

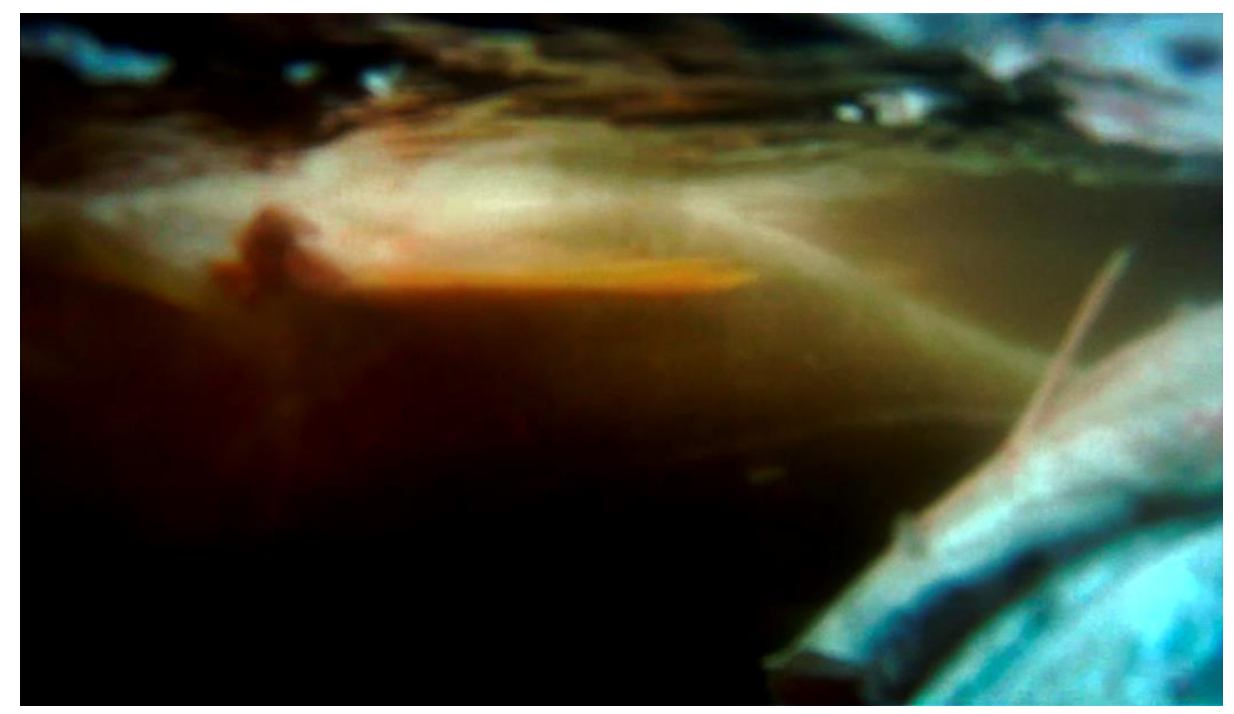

Fig. 4. Leviathan, Véréna Paravel et Lucien Castaing-Taylor, 2012, photogramme du film. 
Il faut alors souligner ce qui forme peut-être la contrepartie ou le contrepoint de cette extraordinaire, troublante et monstrueuse proximité que l'on vient d'expérimenter avec des poissons mourants : la façon qu'ont les objets du monde filmés par Véréna Paravel et Lucien Castaing-Taylor de sortir du cadre du reconnaissable, quand bien même ils nous sont les plus proches. L'usage de certains régimes de monstration, comme le très gros plan, le flou ou le dérèglement de la mise au point, œuvre à «dénaturer» le représenté en augmentant le rapport d'intimité physique. On s'aventure, peut-être d'une autre manière, ici, à la rencontre de l'étrange étranger, cet autre - audelà ou en-deçà - de la perception, qui m'est intérieur tout en m'étant extérieur, qui m'est familier tout en m'étant étranger ; ce que je connais le plus m'étant aussi le plus étranger - lorsque s'accroît l'intimité. Ainsi en est-il de la peau ${ }^{18}$ humaine cadrée en très gros plan [fig. 5]. Un filament d'algue s'est accroché au bras d'un marin, l'image s'en précise, puis perd la netteté, se dissout dans les pores de la peau, jusqu'au flou tant la caméra est proche du derme et peine à faire le point. Les images en très gros plan se déplacent sur divers bras, diverses peaux, diverses épaules diversement tatouées, jusqu'à faire système avec les poissons précédemment filmés dans le bac de triage, et la manière dont nous partagions leur existence. Filmer les hommes comme des poissons. La caméra remonte vers un visage. Apparaissent des rides, des yeux, un fragment de profil, un morceau de visage, filmé de tellement près qu'il est méconnaissable : qu'il semble n'avoir plus rien d'humain [fig. 6]. Sans compassion ni complaisance, consciencieusement égalisante, la caméra se donne pour tâche de scruter les surfaces du monde. Que celles-ci soient humaines, animales, vives, inertes, élémentales, est puissante et déstabilisante l'impression que tout est filmé de la même manière, que tout se prolonge et s'entretisse, que nous plongeons au cœur du maillage. Les gouttes de sueur qui glissent sur le visage de l'homme se prolongent dans le ruissellement de gouttes d'eau du plan suivant, en extérieur jour, sur le pont, tandis que la musique metal qui résonne lors d'un moment de détente dans le carré du bateau se fond dans les bruits mécaniques des engins qui reprennent le dessus dans le plan suivant.

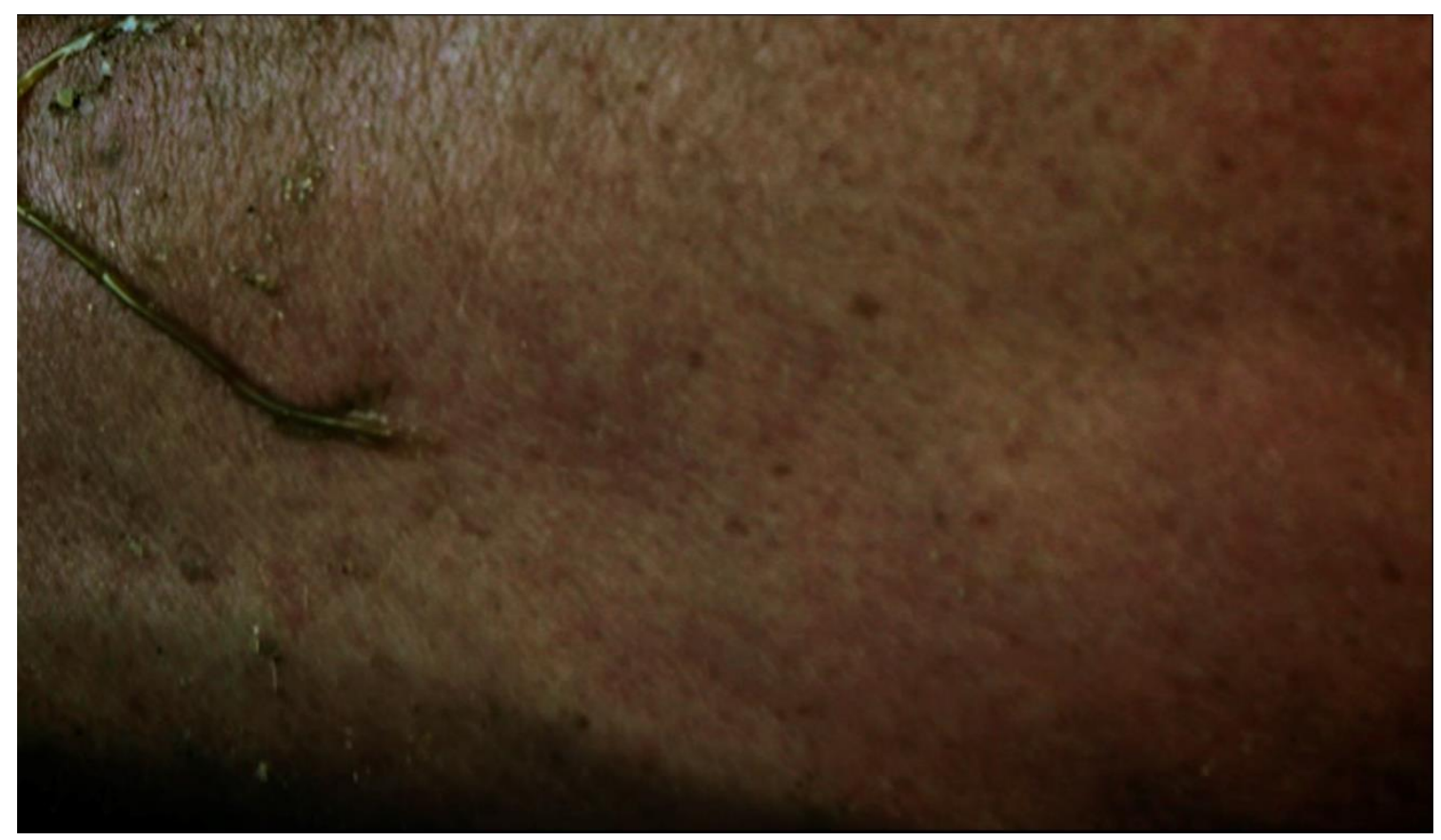

Fig. 5. Leviathan, Véréna Paravel et Lucien Castaing-Taylor, 2012, photogramme du film.

\footnotetext{
18 J'écris « de la peau », et non plus " une personne », tout en sachant très bien que cette peau est celle de l'un des pêcheurs ; je peux certes la rattacher à un individu existant, mais, à l'écran, elle s'autonomise en pure surface.
} 


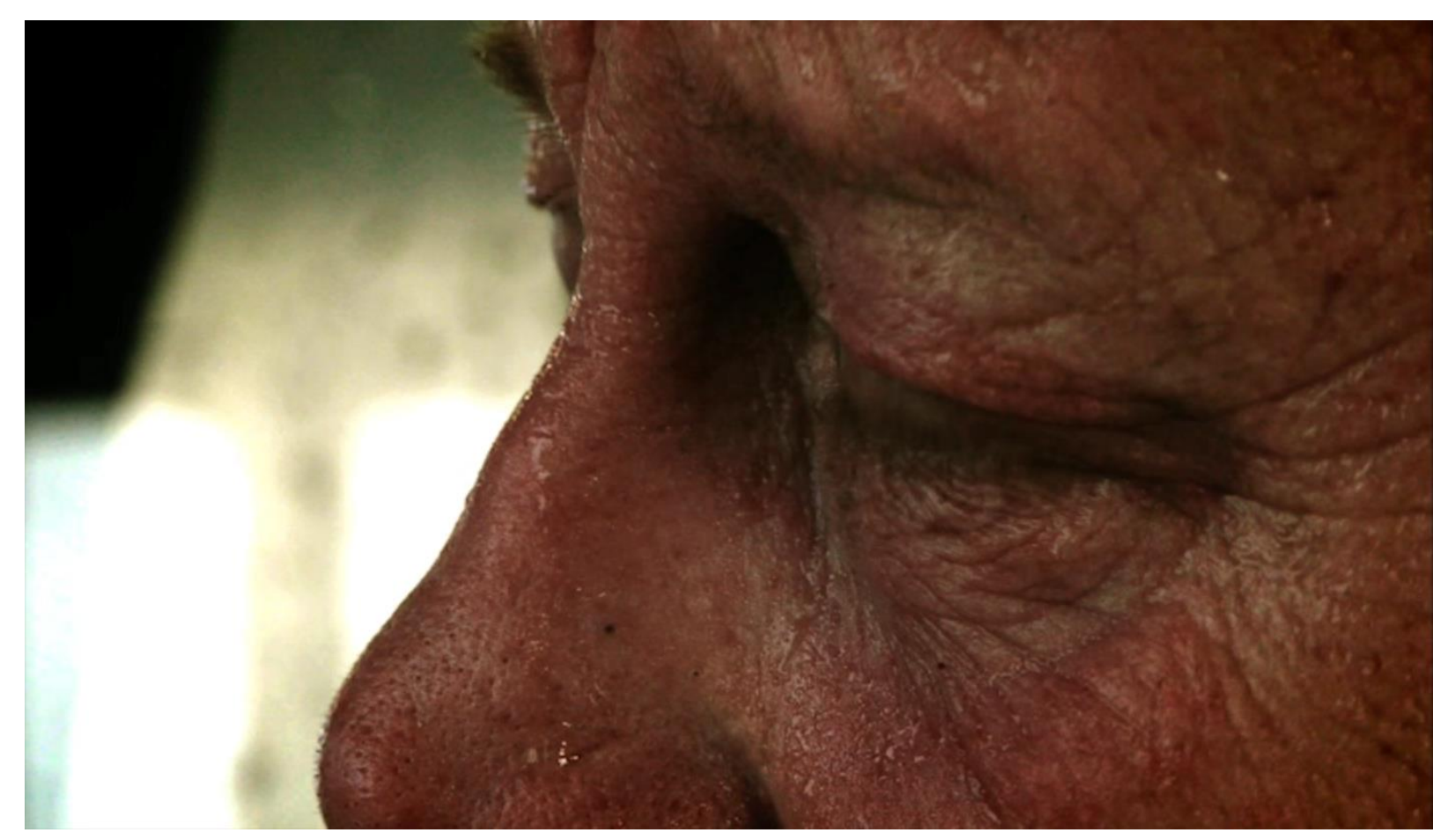

Fig. 6. Leviathan, Véréna Paravel et Lucien Castaing-Taylor, 2012, photogramme du film.

L'image d'un marin sous la douche prolonge cette sensation. Le plan, orange, flouté, se tient au seuil de la perception [fig. 7], dans une continuité sonore et matériologique avec les images précédemment aperçues : le ruissellement de l'eau fait de cet homme se nettoyant du sang et du jus d'entrailles des poissons l'écho ou le fantôme du poisson lui-même, préalablement rincé au tuyau pour le débarrasser des mêmes fluides corporels. Alors, comme les restes indésirables des poissons rejetés dans la mer par les écoutilles, nous replongeons dans l'océan, plus profond que nous n'étions encore allés. L'image outremer, colourfield presque homogène, n'est plus traversée par les mouvements désordonnés de la surface. Les sons qui nous parviennent sont déformés par l'épaisseur de l'eau, sa nature phonique particulière. Puis on distingue un filin, que la caméra suit, descendant jusqu'au filet. Le fond de l'eau boucle sur le bac à douche.

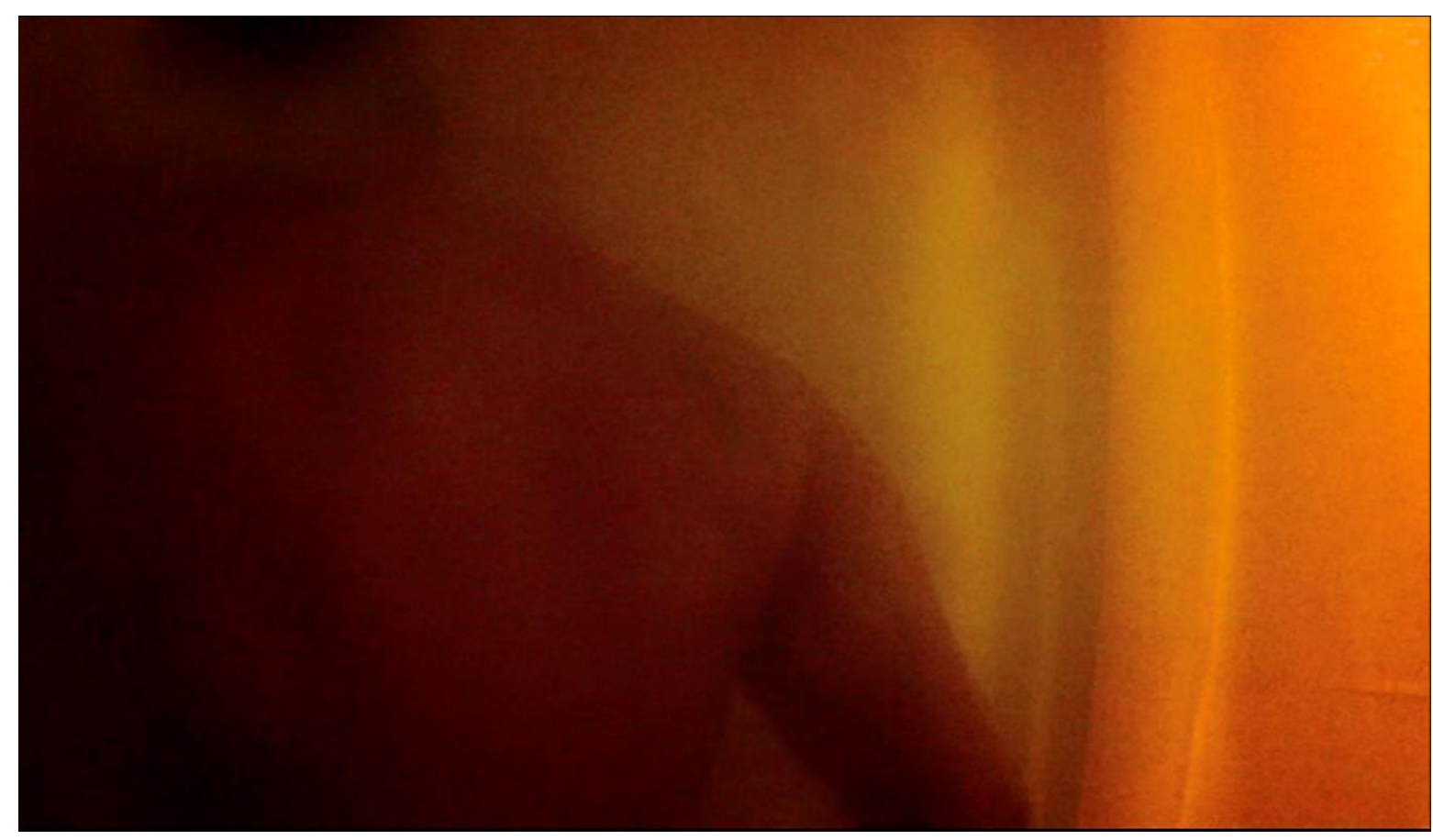

Fig. 7. Leviathan, Véréna Paravel et Lucien Castaing-Taylor, 2012, photogramme du film. 


\section{Une éthique du sonore}

Leviathan se donne aussi comme une expérience sonore inédite. Ernst Karel, le compositeur, a travaillé à partir des sons en prise directe captés sur le bateau à créer une partition qui se tient au seuil du musical, entre le post-rock, le grunge et le heavy-metal. Comme l'expliquent les réalisateurs, l'«intensité sensorielle de la vie à bord est aussi acoustique que visuelle ${ }^{19}$ ». L'omniprésence du moteur du bateau, les différences de régime des turbines, les grincements des poulies pendant la remontée des filets, les sirènes qui annoncent les levées, les voix des hommes, les cris des mouettes, les sons visqueux de la masse des poissons que l'on déverse, les craquètements des coquilles Saint-Jacques que l'on trie, enfin, les sons liquides, multiples, sourds comme brillants de l'océan, constituent l'étendue de la gamme sonore présente dans le film. Le travail de la bandeson dénote d'une éthique du cadrage sonore : d'une conception égalitaire du traitement du son qui distribue à tous les étants leur part de présence sonore, renonçant au vococentrisme trop fréquent dans le cinéma ethnographique - et dans presque tout le cinéma.

Une séquence montre les pêcheurs, fumant une fois que le travail intense de 4 à 5 heures de tri, d'abattage et de préparation des animaux exécuté à chaque levée de filet commence à s'achever. Leurs visages sont maculés de sang et d'éclaboussures diverses. Un regard rencontre la caméra. Les marins discutent entre eux, mais l'on n'entend pas ce qu'ils se disent. Leur conversation est masquée, effacée par l'attention portée aux cris des mouettes, aux sons produits par les corps des poissons, aux bruits des moteurs ou des vagues. Dans ce continuum sonore, tous sont traités à la même enseigne. L'éthique du sonore dans Leviathan consiste à donner à chaque entité sa présence dans la composition d'ensemble, ce qui, comme le suggère Mathias Kusnierz, peut mener à une forme de « [mise] en crise [des] normes de perception et [des] discours anthropocentrés ${ }^{20}{ }$.

Mais, de façon plus subtile encore, le son dans Leviathan passe par d'autres régimes sensibles que le sonore, si je puis dire. De nombreux plans sous-marins, à la limite de l'abstraction mais à la grande pictorialité, nous invitent à une expérience tachiste, parfois à la limite du visible. Ils font penser à des images produites par des techniques d'imagerie récentes, comme l'échographie ou le sonar, qui usent de la mesure de la propagation des sons dans l'eau ou les tissus pour procéder à une traduction visuelle de l'objet renvoyant les ondes sonores ou les ultrasons. Ces procédés, si on les pense en termes de maillage, renvoient tout aussi bien aux régimes de perception des animaux marins (l'écholocalisation de certains cétacés), qu'aux instruments technologiques des bateaux qui leur permettent, par un procédé mimé sur celui des animaux, de repérer les bancs de poissons dans la mer. L'échographie, qui nous permet de nous assurer de la bonne croissance des embryons, nous vient de la même technologie létale que celle qui permet de repérer les sous-marins ennemis en temps de guerre, ou de procéder, par la pêche intensive, à l'épuisement des ressources sous-marines. Alors, à s'approcher au plus près des jonctions du maillage, on ne perçoit plus ni contours ni ordre ontologique.

\footnotetext{
19 " Leviathan, un film fou signé Véréna Paravel et Lucien Castaing-Taylor », op.cit.

${ }^{20}$ Mathias KUSNIERZ, « L'audio-vision documentaire au risque de l'indéchiffrable : le bruit visuel et sonore dans Leviathan de Lucien Castaing-Taylor et Verena Paravel », in « Bruits », L'Autre Musique, n4, mars 2016, [En ligne], URL = [http://lautremusique.net/lam4/funambule/l-audiovision-documentaire-au-risque-de-l-indechiffrable.html], consulté le 11 août 2019.
} 
Comme le note Cyril Neyrat dans un article intitulé Le sang des poissons, la beauté du monstre, «Leviathan sidère en surface par la force du "jamais vu", mais émeut par le sentiment de "déjà vu" remontant des profondeurs du temps ${ }^{21} \gg$. Le film s'inscrit dans une histoire des formes, dont il se nourrit en sollicitant - parfois en creux, parfois en plein -, certaines de ses aventures constituées. Leviathan s'inscrit dans la vaste histoire du cinéma, entendue comme « conquête du sensible liée à une aventure de la visibilité ${ }^{22} »$. Il rappelle également les avant-gardes du cinéma français des années 1920, dont les recherches investiguaient la dimension aqueuse de l'expérience cinématographique (Epstein, Grémillon). Il sollicite, tout autant, l'«histoire longue de la transformation des gens de la mer en images photographiques et cinématographiques - David Octavius Hill, Robert Adamson, Robert Flaherty, John Grierson ${ }^{23}$ », entrecroisant une archéologie de l'océan à une archéologie de l'image. La citation du Livre de Job en ouverture du film, l'usage du latin au générique ${ }^{24}$, la référence à l'œuvre de Melville, entretissent des temps archaïques, mythologiques ou apocalyptiques, à ce temps qui se déroule différemment en mer : comme le soulignent les réalisateurs, " on se retrouve dans une temporalité étirée qui nous ramène à nos origines, ainsi qu'à notre $\operatorname{fin}^{25} \gg$.

Le travail de post-production, notamment le travail chromatique d'étalonnage, s'est construit autour de la sollicitation d'œuvres de Goya, de Bruegel, de Bosch ou de Turner. Enfin, c'est, bien entendu, la référence à la nature morte que le film revisite, en nous donnant l'impression d'assister à une vanité qui se composerait puis disparaîtrait d'elle-même, touchant du doigt, par les moyens de l'image animée, le ressort le plus intime de la nature morte. Un très beau plan sur une tête de poisson appelle une stratification d'images. Des restes, coquilles, étoiles de mer, têtes de raies, sont balayés par les mouvements du bateau, entrent dans le champ, puis disparaissent par l'écoutille. Le film est ainsi traversé par la peinture [fig. 8 et 9], et laisse ainsi percevoir, à travers un éco-système des images, ce que peut être et ce que peut faire une écologie des formes.

Enfin, «comme si Leviathan était lui-même une sorte de mer recelant des trésors cachés dans les interstices des images ${ }^{26} \gg$, le film a donné lieu à un certain nombre d'œuvres, d'installations, et de films dérivés du film original, qui le transforment en univers étendu ${ }^{27}$.

\footnotetext{
${ }^{21}$ Cyril NeYRAT, Le sang des poissons, la beauté du monstre, op. cit.

${ }^{22}$ Id.

${ }^{23}$ Comme le confient les réalisateurs eux-mêmes, in "Leviathan, un film fou signé Véréna Paravel et Lucien Castaing-Taylor ", op.cit.

${ }^{24}$ Le générique de fin indique, en latin, le nom des espèces animales, entremêlé aux noms des protagonistes humains du film, révélant, là encore, cette attitude d'égale attention à l'égard du vivant.

${ }^{25}$ « Leviathan, un film fou signé Véréna Paravel et Lucien Castaing-Taylor », Ibid.

${ }^{26}$ « Leviathan, un film fou signé Véréna Paravel et Lucien Castaing-Taylor », op. cit..

${ }^{27}$ Ce projet plus large s'intitule « Canst Thou Draw Out Leviathan with a Hook? ».
} 


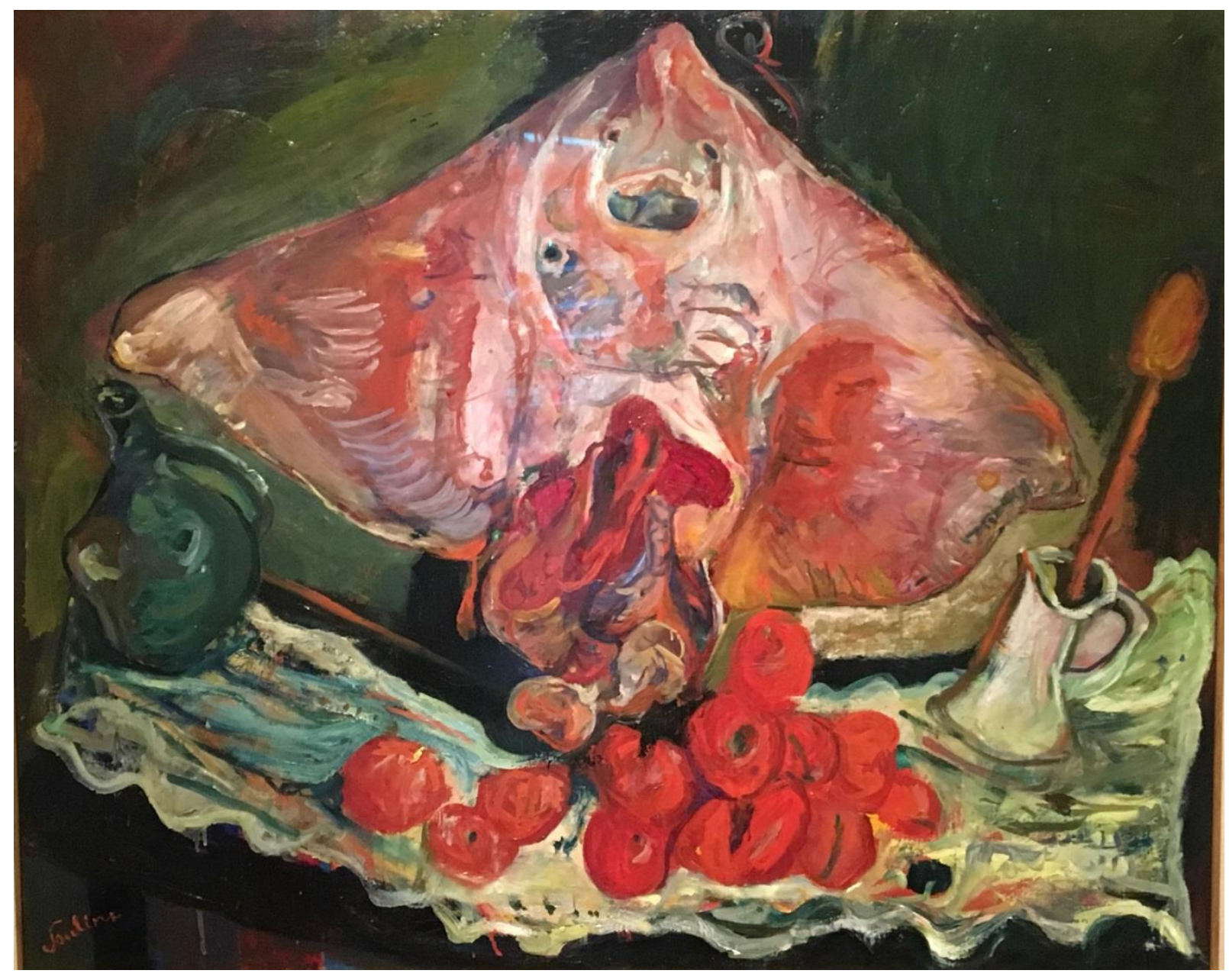

Fig. 8. Chaïm Soutine, La Raie, huile sur toile, vers 1923, New-York.

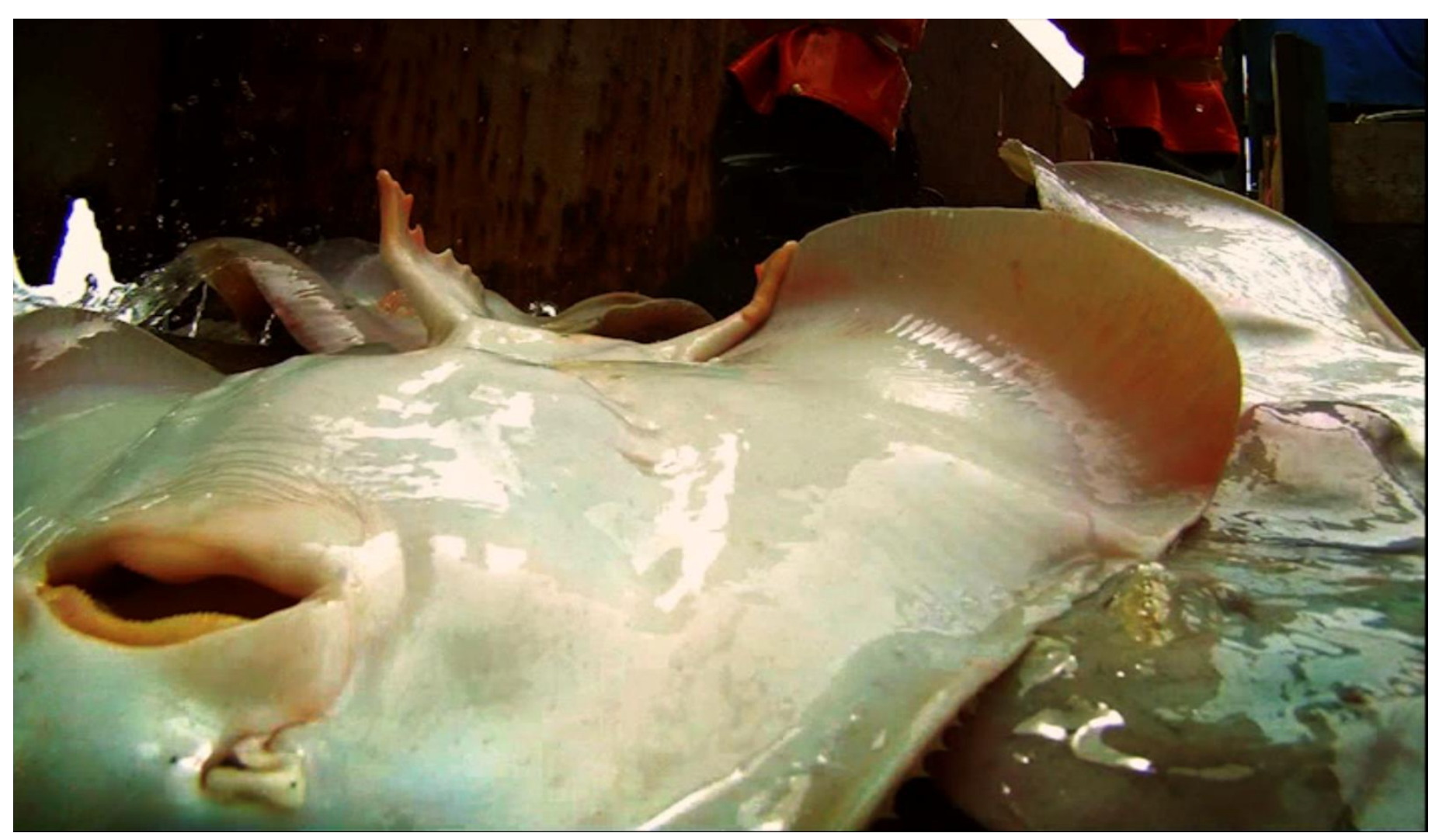

Fig. 9. Leviathan, Véréna Paravel et Lucien Castaing-Taylor, 2012, photogramme du film. 
Dans La Pensée écologique, Timothy Morton souligne, à de nombreuses reprises, la positivité de l'art dans le projet écologique : d'abord parce que «tout art [...] intègre l'environnement jusque dans sa forme $e^{28} »$, tel le poème dont la disposition en paragraphes, dans la page, implique une façon de faire milieu, d'être en lien, de façon multiple. Plus largement, Morton établit le « caractère écologique de tout art ", parce que, fondamentalement, tout art se constitue de matériaux, et qu'il existe dans le monde. Ensuite, de façon assez inattendue a priori (cette dimension rencontre le projet de la dark ecology, partie sombre de l'écologie, théorisée par T. Morton, qui prend en considération l'ironie, la laideur et l'horreur de l'écologie ${ }^{29}$ ), pour les facultés de l'art à accomplir un travail négatif: «L'art peut nous aider parce que c'est un lieu, dans notre culture, qui a trait à l'intensité, la honte, l'abjection, la perte ${ }^{30} \gg$. Enfin, l'importance de (l'étude de) l'art pour la pensée écologique se signale par sa faculté heuristique, par sa capacité à saisir et à exprimer un « réel » dont les mots peinent à se saisir : «L'étude de l'art est importante parce que l'art donne parfois voix à ce qui est partout ailleurs indicible, soit temporairement - nous trouverons les mots un jour -, soit intrinsèquement - les mots sont impossibles ${ }^{31} »$. En l'occurrence, ici, «la majesté, l'horreur, la puissance écrasante de la mer $^{32} \gg$.

\section{Auteur}

Sophie Lécole Solnychkine est MCF en Arts plastiques à l'Université Toulouse - Jean Jaurès, où elle enseigne l'esthétique et la philosophie de l'art. Elle est responsable de l'équipe de recherche SEPPIA (Savoirs, praxis et poḯtique en arts), composante du LARA-SEPPIA (EA 4154). Spécialiste du paysage dans les arts visuels (peinture, cinéma), elle développe ses travaux de recherche au croisement de l'histoire des formes et de l'histoire des idées, depuis les sites théoriques que constituent les images cinématographiques et picturales. Elle a fondé et co-dirige (avec Patrick Barrès) la revue Gradalis, consacrée aux expériences paysagères de terrain (textes, images, sons), dont le deuxième numéro, Visions minérales, est paru à l'automne 2019 aux Éditions Passage(s). Son premier ouvrage personnel, Asthetica antarctica. The Thing de John Carpenter paraît aux Éditions Rouge Profond en novembre 2019.

\footnotetext{
${ }^{28}$ Timothy MORTON, op.cit., p. 26.

${ }^{30}$ Ibid., p. 27.

${ }^{31}$ Timothy MORTON, op. cit., p. 30-31.

32 " Leviathan, un film fou signé Véréna Paravel et Lucien Castaing-Taylor », op. cit.
}

${ }^{29}$ " J'explore la possibilité d'une nouvelle esthétique écologique : l'écologie sombre (dark ecology). L'écologie sombre introduit dans le penser écologique de l'hésitation, de l'incertitude, de l'ironie et de l'attention. La forme de l'écologie sombre, c'est le film noir. Dans un film noir, le narrateur débute son enquête de manière $a$ priori objective sur une situation qui lui est $a$ priori extérieure, avant de découvrir qu'il est impliqué. Le point de vue du narrateur se teinte alors de désir. II n’y a pas de métaposition à partir de laquelle nous puissions faire des déclarations écologiques ", in Timothy MORTON, Ibid., p. 37. 